\title{
A NEW CLASS OF SPECTRAL OPERATORS ${ }^{1}$
}

BY H. H. SCHAEFER

Communicated by W. S. Massey, October 14, 1960

Let $X$ be an ordered (=partially ordered) complex Banach space (cf. $[3, \S 6]$ ). The positive cone $K=\{x: x \geqq 0\}$ in $X$ is normal if there exists $\gamma>0$ such that $\|x+y\| \geqq \gamma\|y\|$ for all $x, y \in K$. We say that a complex $B$-algebra $A$ (with unit $e$ ) is ordered if the underlying $B$-space is ordered with $K$ closed and normal, and if $K$, in addition, has these properties: (i) $e \in K$; (ii) $a \in K, b \in K$ and $a b=b a$ imply $a b \in K$ (cf. $[3, \S 11]$ ). We shall write, as usual, $x \leqq y$ (or $y \geqq x$ ) for $y-x \in K$, and $[x, y]=\{z: x \leqq z \leqq y\}$. The term "semi-complete" stands for "sequentially complete." For any $a \in A, \sigma(a)$ denotes the spectrum of $a$. A function $\mu$ from the Borel sets of the real line $R$ into $A$ is a Borel measure if $\mu$ is countably additive, i.e., if $\mu\left(\cup_{1}^{\infty} \delta_{n}\right)=\sum_{1}^{\infty} \mu\left(\delta_{n}\right)$ converges in $A$ for an arbitrary sequence $\left\{\delta_{n}\right\}$ of mutually disjoint Borel sets.

Theorem 1. Let $A$ be an ordered B-algebra, such that $[0, e]$ is weakly semi-complete. Let $c_{1} e \leqq a \leqq c_{2} e$ where $c_{1}, c_{2} \in R$. Then $\sigma(a) \subset\left[c_{1}, c_{2}\right]$, and there exists an $A$-valued Borel measure $\mu$ such that

$$
a^{n}=\int_{\sigma(a)} t^{n} d \mu, \quad(n=0,1,2, \cdots) .
$$

Moreover, $\mu$ is a homomorphism of the Boolean $\sigma$-algebra of real Borel sets onto a Boolean $\sigma$-algebra of idempotents contained in $[0, e]$, and

$$
f \rightarrow \int_{\sigma(a)} f d \mu
$$

is an order preserving homomorphism of the algebra of bounded Borel functions on $\sigma(a)$, into $A$.

If $A$ is a (Banach) algebra of bounded operators on a $B$-space $X$, then an $a \in A$ satisfying the assertions of Theorem 1 is a (scalar type) spectral operator in the sense of Dunford [1]. We obtain from Theorem 1:

THEOREM 2. Let $A$ be an ordered B-algebra of operators on a weakly semi-complete $B$-space $X$. Then every operator a which is contained in the real linear hull of $[0, e]$ is a scalar type spectral operator, $a=\lambda \lambda d \mu$, with real spectrum $\sigma(a)$, and $\mu$ is a spectral measure with values in $[0, e]$.

1 Research sponsored by the Office of Ordnance Research, U. S. Army. 
CoROLlaRy. Let $a \in A$. If the convex cone (of vertex 0 ) spanned by the set $\left\{a^{m}(e-a)^{n}: m, n=0,1,2, \cdots\right\}$ is normal, then $a$ is a scalar type spectral operator with real spectrum.

Let $X$ be a complex Hilbert space of arbitrary dimension; the algebra $A$ of bounded operators on $X$ is ordered with respect to the familiar positivity notion for Hermitian elements of $A$; since every Hermitian operator is in the real linear hull of $[0, e]$, the spectral theorem for bounded Hermitian operators is a special case of Theorem 2. Theorem 2 can be extended to elements $a+b i$ where $a b=b a$ and $a, b$ are both in the real linear hull of $[0, e]$. Thus the spectral theorem for (bounded) normal operators in Hilbert space is a consequence of Theorem 2. If $A$ is an algebra of operators on an arbitrary Banach space, the analog of the cone of positive Hermitian operators is the cone $K_{\mathrm{s}}$ spanned by finite sums of squares; if $K_{\mathrm{s}}$ is normal and weakly semi-complete, all $a \in A$ are scalar type spectral operators with real spectrum. $K_{s}$ has been considered, e.g., in [2]. Another general example can be obtained as follows.

TheOREM 3. Let $X$ be an ordered Banach space whose positive cone $K$ is normal, weakly semi-complete, and generating. ${ }^{2}$ Then the algebra $A$ of bounded operators on $X$ is an ordered $B$-algebra with positive cone $\{a \in A: a K \subset K\}$, and every element in the real linear hull of $[0, e]$ is a scalar type spectral operator such that $\sigma(a) \subset\left[c_{1}, c_{2}\right]$ if $c_{1} e \leqq a$ $\leqq c_{2} e\left(c_{1}, c_{2} \in R\right)$.

It follows from this theorem that there are nontrivial scalar type spectral operators on every weakly semi-complete Banach space. Proofs of the announced results and further results concerning compact operators and unbounded operators will be published elsewhere.

\section{REFERENCES}

1. N. Dunford, $A$ survey of the theory of spectral operators, Bull. Amer. Math. Soc. vol. 64 (1958) pp. 217-274.

2. J. L. Kelley and R. L. Vaught, The positive cone in Banach algebras, Trans. Amer. Math. Soc. vol. 74 (1953) pp. 44-55.

3. H. Schaefer, Halbgeordnete lokalkonvexe Vektorräume. II, Math. Ann. vol. 138 (1959) pp. 259-286.

The University of Michigan

2I.e., $X=K-K$. 